\title{
Application of the Particle Filter in Maneuver Target Turn Tracking Algorithm
}

\author{
Zhenda Lei ${ }^{1}$ \\ ${ }^{1}$ Jiangsu Automation Research Institute, Lianyungang 222061, China \\ ${ }^{1}$ laozhanshi124@163.com
}

Keywords: Non-linear; Non-Gausss;Particle Filtering; Uncertainties;

\begin{abstract}
The non-linear target-tracking method has been extensive researched to solve turn maneuver target. Particle filter is suitable for any non-linear, non-Gaussian system that could be represented with many kinds of turn maneuver, Good Methods are needed in Maneuver Tracking. Particle filter is demonstrated that it has better performance when compared with the Extended kalman filter to target tracking. when used in tracking algorithm, the new filter yields improved performance in the case of model uncertainties. The simulation results indicate that manure tracking algorithm can maintain track under severe correlates maneuvers when using Particle filter which is better than Extended kalman filter.
\end{abstract}

\section{Introduction}

With the rapid development of modern science and technology, speed and maneuverability of various high speed maneuvering aircraft has become increasingly high, such as modern anti-ship missile is mobile, there are: climbout, $\mathrm{S}$ maneuver, reentry flight, end winding subduction and spiral maneuver [1]. Particle filter (Filter PF, Particle) has become a hot and effective method to study the optimal estimation of nonlinear and non Gauss dynamic systems [3]. This paper introduces the particle filter algorithm for nonlinear systems, the particle filter algorithm is better than the extended Kalman filter (EKF) is more in line with the actual situation of [4] [5] [6] tracking filter, can achieve good tracking performance. For high speed maneuvering target tracking simulation results show that compared with using the extended Kalman filter algorithm, this algorithm improves the processing ability of turn targets, can obtain good tracking effect.

\section{Maneuvering Target Turning Maneuver Model}

Maneuvering target turning motion is a kind of complicated target maneuver, and the target trajectory is usually a kind of circle. Set a target at a certain point, as shown in Figure 1. The target variable includes the position $\mathrm{x}$ and $\mathrm{y}$, velocity $v$, the direction of the velocity $\phi$, the acceleration of the acceleration $a_{n}$ and the tangential acceleration $a_{t}$.

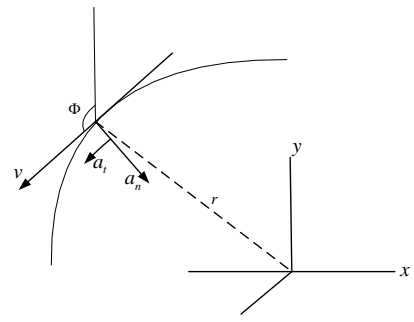

Fig. 1 Target Motion Model

\section{Particle Filter}

Assuming the state sequence of the actual system $\left\{x_{k}, k \in N\right\}$, which $K$ is the time sequence 
label, the state vector $x_{k} \in R^{n_{x}}$ that represents the time $K$ label is the state vector, which is the dimension of the state vector $n_{v} \in N$. The transition probability between states is:

$$
x_{k}=f_{k}\left(x_{k-1}, v_{k-1}\right)
$$

The nonlinear state transfer function of the system is $f_{k}: R^{n_{x}} \times R^{n_{x}} \rightarrow R^{n_{x}}$, $\left\{v_{k-1}, k \in N\right\}$ independent of the dimension $n_{v} \in N$ of the same distributed noise sequence ${ }^{v_{k-1}}$, or it can be described by the form of the probability matrix:

$$
p\left(x_{k} \mid x_{k-1}\right)
$$

The observed sequence $\left\{z_{k}, k \in N\right\}$ of the system is the observation vector, which $z_{k} \in R^{n_{x}}$ represents the time label as the time $K$, and the dimension of the observation vector $n_{x} \in N$. The relationship between the system and the system is:

$$
z_{k}=h_{k}\left(x_{k}, n_{k}\right)
$$

$h_{k}: R^{n_{x}} \times R^{n_{x}} \rightarrow R^{n_{x}}$ is a system of nonlinear observation function, $\left\{n_{k}, k \in N\right\}$ is an independent of the same distribution of noise sequences, $n_{n} \in N$ is $n_{k}$ the dimension, or can also be described in the form of a probability matrix:

$$
p\left(z_{k} \mid x_{k}\right), k \geq 0
$$

The most important problem of the state space model is to estimate the state $x_{k+1}$ based on the observation vector $z_{1: k}=\left(z_{1}, z_{2}, \cdots, z_{k}\right)$ and the initial distribution $p\left(x_{0}\right)$. Many of the problems in time series, such as the construction of likelihood parameter estimation and prediction calculation, interpolation and stationary or non-stationary time series can be solved by state estimation, for three different values $i$ of the state estimation problem is divided into three different types $i=0, i>0, i<0$, are called filtering, prediction and smoothing.

$$
\begin{aligned}
& z_{1: k}=\underset{p\left(x_{0}\right)}{\left(z_{1}, z_{2}, \cdots, z_{k}\right) \Rightarrow p\left(x_{k+i} \mid x_{k}\right),} \\
& i=0 \text {; filter } \\
& i>0 \text {; perdict } \\
& i<0 \text {; smooth }
\end{aligned}
$$

\section{Particle Filter Algorithm}

Particle filter is a sequential Monte Carlo filtering method, and its essence is to use a series of random samples, that is, particle, to replace the state of the posterior probability distribution. When the number of particles becomes large enough, the state can be obtained by the random sampling method. At the same time, the advantages of particle filter is also reflected in the complexity and accuracy of it only with the number of particles, and the particle filter algorithm itself is independent, and the state space dimension, this particle filter it will not increase the dimension of the state space of the performance decrease or increase of complexity.

Specific particle filter algorithm is as follows:

$$
\begin{aligned}
& {\left[\left\{x_{k}^{i}, w_{k}^{i}\right\}_{i=1}^{N}\right]=P F\left[\left\{x_{k-1}^{i}, w_{k-1}^{N}\right\}, z_{k}\right]} \\
& \bullet F O R i=1,2, \cdots, N \text { do } \\
& \text { - FOR } i=1,2, \cdots, N \text { do } \\
& x_{k}^{i} \sim q\left(x_{k} \mid x_{k-1}^{i}, z_{k}\right) \text { SAMPLE } \\
& \text { for } w_{k}^{i} \propto \frac{p\left(z_{k} \mid x_{k}^{i}\right) p\left(x_{k}^{i} \mid x_{k-1}^{i}\right)}{q\left(x_{k}^{i} \mid x_{0: k-1}^{i}, z_{1: k}\right)}
\end{aligned}
$$


- END FOR

CALCULATE: $t=\sum_{i=1}^{N} w_{k}^{i}$

- $F O R i=1: \quad N$ do

$$
\text { NORMALIZE: } w_{k}^{i}=\frac{w_{k}^{i}}{t}
$$

- END FOR

$\hat{N}_{\text {eff }}=\frac{1}{\sum_{i=1}^{N}\left(w_{k}^{i}\right)^{2}} \quad C A L C U L A E \hat{N}_{\text {eff }}$

- IF $\hat{N}_{\text {eff }}<N_{T}$ then

$$
\text { RESAMPLE }
$$

$$
\left[\left\{x_{k}^{i}, w_{k}^{i},-\right\}_{i=1}^{N}\right]=R E S A M P L E\left[\left\{x_{k}^{i}, w_{k}^{i}\right\}_{i=1}^{N}\right]
$$

- END IF

\section{Simulation Results}

Monte-Carlo simulation is used to verify the effectiveness of the algorithm. The simulation times are 100 times. Particle number $\mathrm{N}=200$. Considering the tracking of maneuvering targets, this paper also compared using particle filter and extended Kalman filter algorithm for maneuvering motion model to simulate the typical turn, sampling period $T_{s}=1 s$, experiment with a turning maneuver missile as an example, the starting point is assumed to be m (25000 10000): 1-20s, the trajectory of uniform linear motion, speed for $707 \mathrm{~m} / \mathrm{s}$, for $21-40$ s to target direction angle $9^{\circ} / \mathrm{s}$, counterclockwise for turning motion, 41-60s linear movement, 61-80s target to along the counterclockwise turning motion $9^{\circ} / s$, 81-100s linear motion to attack the ship. Process noise $v_{k-1} \sim N(0,5)$, observation noise $w_{k} \sim N(0,5)$.

By means of statistical root mean square error (RMSE) to represent the estimation of the state of the target, the root mean square of the position and speed of the two filtering algorithms are shown in Table 1.

Table 1 Root mean square statistics of two kinds of filtering algorithms

\begin{tabular}{c|cc}
\hline & $E K F$ & $P F$ \\
\hline position $\mathrm{m}$ & 5.7312 & 1.2548 \\
speed $\mathrm{m} / \mathrm{s}$ & 2.6415 & 1.0415
\end{tabular}

Figure 2 and figure 3 are presented in this paper, the particle filter algorithm, based on the extended Kalman filter algorithm in the case of turning maneuver simulation results.

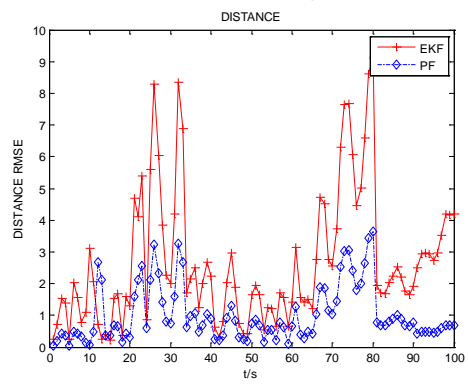

Fig2 Comparison of the tracking results of two filtering algorithms 


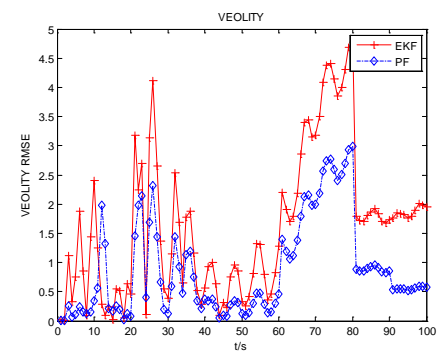

Fig3 Comparison of filtering algorithms for velocity tracking

The simulation results show that the filtering error of the two algorithms is quite different, which indicates that the divergence phenomenon occurs when the target of the high speed and high maneuver is turning maneuver, and the algorithm can restrain the divergence. This is because the particle filter method is better adapted to the change of the tracking when it is in the process of maneuver, the algorithm always keeps track of the target state.

\section{Conclusions}

This paper introduces particle filter for nonlinear and non Gauss model of target tracking, compared with the extended Kalman filter to get a more satisfactory tracking performance, enhance the occurrence of mutation of tracking capability, and tracking of maneuvering target performance generally remain unchanged. The simulation results show that this algorithm significantly improves the performance of the tracking maneuvering target, and has good application prospects compared with the algorithm using the extended Kalman filter.

\section{Reference}

[1] Liu Lili. Study on the attack mode of anti ship missile [J]. Shanghai aerospace, third phase of 1999

[2]Kalman R.E.A, New Approach to Linear [2] Filtering and Prediction Problems[J].Transaction of ASME-Journal of Basic Engineering pp.35-45,, Match, 1960

[3]Arnaud Doucet Nando, de Freitas Neil, Gordon. Sequential Monte [6] Carlo In Practice[M].New York:Spinger-Verlag.2001:9 19

[4] Liu Xin, Guo Yunfei, Xue Anke. Comparison of [J]. mathematics and computer engineering several filter tracking performance. 2013 third, No.3 Vol.41 391

[5] Calman Hu Yongchang and the application of particle filter in GPS multipath estimation [J]. Electronic design engineering. 2013.2, Vol.21, No.4

[6] Zhang Meng, Chen Ken, Li Na, Hui Ming. Optimization of tracking mode between [J] Kalman filter and particle filter. Computer engineering and applications, 2012, 48 (36) 